Rüdiger Kipke

Das armenisch-aserbaidschanische Verhältnis und der Konflikt um Berg-Karabach 
Rüdiger Kipke

\author{
Das armenisch- \\ aserbaidschanische \\ verhältnis \\ und der Konflikt \\ um Berg-Karabach
}


Bibliografische Information der Deutschen Nationalbibliothek

Die Deutsche Nationalbibliothek verzeichnet diese Publikation in der

Deutschen Nationalbibliografie; detaillierte bibliografische Daten sind im Internet über

$<$ http://dnb.d-nb.de> abrufbar.

\section{Auflage 2012}

Alle Rechte vorbehalten

(c) VS Verlag für Sozialwissenschaften | Springer Fachmedien Wiesbaden GmbH 2012

Lektorat: Dorothee Koch

VS Verlag für Sozialwissenschaften ist eine Marke von Springer Fachmedien.

Springer Fachmedien ist Teil der Fachverlagsgruppe Springer Science+Business Media.

www.vs-verlag.de

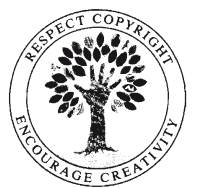

Das Werk einschließlich aller seiner Teile ist urheberrechtlich geschützt. Jede Verwertung außerhalb der engen Grenzen des Urheberrechtsgesetzes ist ohne Zustimmung des Verlags unzulässig und strafbar. Das gilt insbesondere für Vervielfältigungen, Übersetzungen, Mikroverfilmungen und die Einspeicherung und Verarbeitung in elektronischen Systemen.

Die Wiedergabe von Gebrauchsnamen, Handelsnamen, Warenbezeichnungen usw. in diesem Werk berechtigt auch ohne besondere Kennzeichnung nicht zu der Annahme, dass solche Namen im Sinne der Warenzeichen- und Markenschutz-Gesetzgebung als frei zu betrachten wären und daher von jedermann benutzt werden dürften.

Umschlaggestaltung: KünkelLopka Medienentwicklung, Heidelberg

Satz: Jens Ossadnik

Gedruckt auf säurefreiem und chlorfrei gebleichtem Papier

Printed in Germany

ISBN 978-3-531-18484-5 


\section{Inhaltsverzeichnis}

Vorwort...................................................................................................................

1 Historische Wurzeln des armenisch-aserbaidschanischen Konflikts um Berg-Karabach ............................................................................................ 9

1.1 Gründung des Feudalstaats Karabach ................................................. 9

1.2 Das Khanat Karabach als Vasall des Russischen Reiches .................... 10

1.3 Die Annexion des Khanats Karabach ................................................. 13

1.4 Zaristische Kolonialherrschaft in Aserbaidschan................................... 17

1.4.1 Neugliederung und Bevölkerungspolitik ................................. 17

1.4.2 Zustrom von Armeniern aus Persien und dem

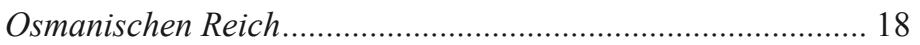

1.4.3 Die Folgen: Instabilität und Krieg........................................... 21

2 Nach dem Ende des zaristischen Imperiums (1917-1923) ...................... 23

2.1 Fortsetzung der Gewalt .................................................................. 23

2.2 Territorialer Bestand der Volksrepublik Aserbaidschan ..................... 24

2.3 Die Konferenz von Batum: Abtretung von Eriwan an Armenien ...... 25

2.4 Errichtung des Generalgouvernements Berg-Karabach ...................... 26

2.5 Regelung von Territorialfragen unter Sowjetherrschaft...................... 27

2.5.1 Gründung der Sowjetrepublik Aserbaidschan ........................ 27

2.5.2 Moskau und der armenisch-aserbaidschanische Konflikt ........ 28

2.5.3 Schulterschluss zwischen Armenien und Sowjetrussland.......... 30

2.5.4 Wechsel in Strategie und Taktik............................................ 32

2.5.5 Gründung der Sowjetrepublik Armenien ............................... 33

2.5.6 Bildung des Autonomen Gebiets Nachitschewan als Teil Aserbaidschans ................................................................... 33

2.5.7 Abtretung von Sangesur an Armenien ........................................ 34

2.5.8 Bildung des Autonomen Gebiets Berg-Karabach innerhalb Aserbaidschans .................................................................... 35 
3 Der Konflikt um Berg-Karabach in seiner latenten Phase (1923-1987) 41

3.1 Zur Einleitung: Sowjetföderalismus und Nationalitätenpolitik 41

3.2 Die Büchse der Pandora: Die Instrumentalisierung des Nationalismus 44

3.2.1 Die ,armenische Karte“ in der sowjetischen Türkei-Politik 44

3.2.2 Schriftwechsel zwischen Arutjunow und Bagirow ................... 49

3.2.3 Eine Gedenkveranstaltung und ihre Folgen .......................... 52

3.2.4 Kontroversen um die Geschichte ........................................... 53

3.3 Die Petitionen der 1960er Jahre - Behauptungen und Tatsachen ....... 58

3.4 Fortdauer der Spannungen in den 1970er Jahren ................................ 62

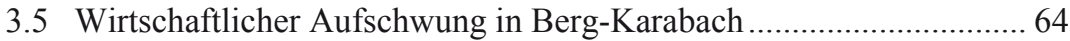

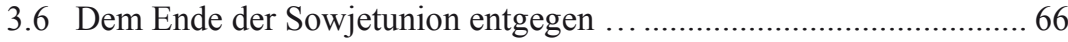

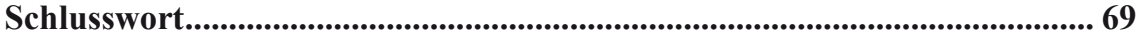

Quellen- und Literaturverzeichnis ..................................................................... 73

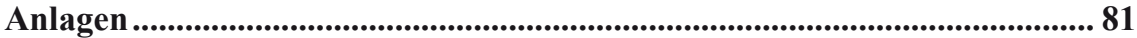




\section{Vorwort}

In den vergangenen zwei Jahrzehnten, seit dem Zusammenbruch der Sowjetunion hat der Kaukasus als geopolitischer Raum verstärkte Aufmerksamkeit in der westlichen Öffentlichkeit gefunden. Die Öl- und Gasreserven an den Küsten des Kaspischen Meeres sind in den Fokus des Interesses gerückt. Zudem haben die politischen Konflikte in dieser Region immer wieder für Schlagzeilen gesorgt. Von Krieg und Gewalt in Tschetschenien und anderen nordkaukasischen Republiken der Russländischen Föderation wird seit Jahren berichtet. Die militärische Auseinandersetzung zwischen Georgien und Russland im August 2008 hat gezeigt, dass die Ereignisse in dieser Region auch den internationalen Frieden bedrohen können.

In der vorliegenden Studie wird ein anderer kaukasischer Brennpunkt behandelt, der Konflikt zwischen Armenien und Aserbaidschan. Er ist im Gegensatz zu den vorher genannten in der öffentlichen Wahrnehmung des Westens kaum existent, obwohl die kriegerischen Auseinandersetzungen um das Gebiet Berg-Karabach in den Jahren unmittelbar vor und nach dem Zusammenbruch der Sowjetunion mit ihren vielen Tausend Toten und zahllosen Verwundeten, mit wechselseitigen Vertreibungen und Fluchtbewegungen $\mathrm{zu}$ gewaltigen Opfern geführt haben. Nachdem bewaffnete Karabach-Armenier und reguläre Truppenverbände Armeniens das aserbaidschanische Gebiet von Berg-Karabach und die angrenzenden Kreise unter ihre Kontrolle gebracht hatten, ist es im Mai $1994 \mathrm{zu}$ einem Waffenstillstand gekommen, der bis heute eine instabile Ruhe garantiert. Seither sind diese Territorien vom übrigen aserbaidschanischen Staatsgebiet faktisch abgetrennt. Die dort beheimateten Aserbaidschaner sind geflohen oder vertrieben worden. Berg-Karabach hat sich am 2. September 1991 für unabhängig erklärt. Das Gebiet wird von niemandem als Staat anerkannt. Auch Armenien verweigert die Anerkennung, bildet aber mit Berg-Karabach eine Wirtschaftsund Währungsunion.

Der Sicherheitsrat der UNO hat mehrere Resolutionen verabschiedet, die das Recht Aserbaidschans auf territoriale Integrität unter Einschluss von BergKarabach bestätigen und zu einer friedlichen Lösung des Konflikts aufrufen. Die Vollversammlung der UNO hat von Armenien vergeblich den vollständigen Abzug seiner Truppen aus den besetzten aserbaidschanischen Gebieten verlangt. Diplomatische Vermittlungsbemühungen wurden auf verschiedenen Ebenen 
unternommen, die bisher ohne Erfolg geblieben sind. Die Brisanz des Konflikts steht außer Frage, kann er doch zur Destabilisierung der ganzen Region führen und internationale Auswirkungen haben.

Der wissenschaftliche Diskurs über den südkaukasisch-kaspischen Raum in den westlichen Ländern wird weitgehend von Historikern getragen, dabei stehen Fragen der Siedlungsgeschichte und der Ethnogenese im Vordergrund. Die herrschende Meinung des Westens folgt der armenischen Position, wonach die Armenier schon im Altertum in diesem Gebiet ansässig waren. Die Auffassung wird in den Ländern geräuschvoll vertreten, in denen die armenische Diaspora besonders stark vertreten und aktiv ist.

Diese Fragen stehen hier jedoch nicht im Zentrum der Erörterung. Die Arbeit befasst sich vielmehr mit den armenisch-aserbaidschanischen Konflikten beginnend mit den armenischen Zuwanderungen im 19. Jahrhundert in den nordaserbaidschanischen Raum. Sie setzt den Schwerpunkt auf die territorialen Auseinandersetzungen zwischen beiden Völkern, die auf unterschiedlichen Ebenen stattfanden. Dabei kommt dem Streit um das Gebiet Berg-Karabach herausragende Bedeutung zu.

Der vorliegende Beitrag ist geeignet, die Debatte um Berg-Karabach um einige neue Aspekte zu bereichern. Es konnte eine Reihe von Archivmaterialien verwertet werden, die bisher in der wissenschaftlichen Öffentlichkeit nicht bekannt waren. Einige der schwer zugänglichen Dokumente, die sich im persönlichen Besitz des Verfassers befinden, sind im Anhang abgedruckt, um sie dem interessierten Leser zugänglich zu machen.

Anlass zum Dank habe ich gegenüber meinem wissenschaftlichen Mitarbeiter, Herrn Rauf Dschafarow, der mit seiner vielfältigen Zuarbeit für das Gelingen des Werkes unverzichtbar war. 